\title{
Organic iso-type pentacene - lead phthalocyanine heterostructures
}

\author{
Ya.I. Vertsimakha, P.M. Lutsyk \\ Institute of Physics, NAS of Ukraine, 46, prospect Nauky, 03680 Kyiv, Ukraine \\ Phone: +380 44-52509 12; fax+380 44-5251589 \\ E-mails:yavertsi@iop.kiev.ua; lutsyk@univ.kiev.ua
}

\begin{abstract}
The photovoltaic properties of organic iso-type heterostructures based on pentacene $(\mathrm{Pn})$ and lead phthalocyanine $(\mathrm{PbPc})$ prepared by thermal deposition at different substrate temperatures $\left(T_{s}\right)$ are investigated. It is shown that, at modulated illumination for $\mathrm{Pn} / \mathrm{PbPc}$ heterostructures prepared at $T_{s}=300 \mathrm{~K}$, the reversal of a sign is observed in photovoltage spectra. The properties of the structures are well described by the Van Opdorp model that indicates the presence of the high surface recombination rate of charge carriers at the interface of heterostructure components. At this, the contribution of heterostructure components to the photovoltage formation can be changed with unmodulated monochromatic additional illumination. In $\mathrm{Pn} / \mathrm{PbPc}$ heterostructures prepared at $T_{s}=370 \mathrm{~K}$, there is no reversal of the photovoltage sign, and the photovoltage is significantly (up to twice) higher in comparison with both heterostructures obtained at $T_{s}=300 \mathrm{~K}$ and separate layers of components ( $\mathrm{Pn}$ and $\mathrm{PbPc}$ ). This is the evidence for the low recombination rate of charge carriers at the interface of $\mathrm{Pn} / \mathrm{PbPc}$ heterostructures prepared at $T_{s}=370 \mathrm{~K}$.
\end{abstract}

Keywords: iso-type heterostructure, photovoltage spectra, lead phthalocyanine, pentacene.

Manuscript received 14.02.07; accepted for publication 24.04.07; published online 19.10.07.

\section{Introduction}

The low efficiency of organic solar cells (SC) is caused by a lot of reasons. One of these reasons is the high series resistance that can be sufficiently decreased for inorganic SC by the formation of $p-p^{+}$and $n-n^{+}$junctions with the help of doping by appropriate dopants [1]. For organic SC, this method of decreasing the series resistance is inefficient and technologically complicated. Therefore, to solve this problem, the use of $p-p^{+}\left(n-n^{+}\right)$ heterostructures (HS) of the iso-type was suggested [2]. These HS can be readily prepared under the same technical conditions simultaneously with obtaining the aniso-type HS. The optimal selection of the components can allow one to widen a spectral region of photosensitivity and to increase the efficiency of the collection of charge carriers, if the concentration of surface states and the recombination rate of charge carriers are low at the interface of HS.

To date, the photovoltaic properties of organic isotype HS is weakly investigated. Therefore, the aim of this work is the study of the photovoltaic properties of iso-type HS based on photosensitive organic semiconductors and the ascertainment of the possibility to prepare HS with low surface recombination rate at the interface for the improvement of the performance of organic SC.

\section{Experimental}

As a basic model object, we selected $p-p^{+}$HS based on photosensitive organic semiconductor pentacene (Pn) that was intensively used for the fabrication of organic $\mathrm{SC}$ [3] and lead phthalocyanine (PbPc), whose conductivity (in the monoclinic modification) is several orders higher [4] than that of $\mathrm{Pn}[5,6]$, and the spectral region of photosensitivity of $\mathrm{PbPc}$ films is considerably wider than that of Pn films [7]. The molecular structures of these materials are presented in Fig. 1. As a consequence of the component selection, the doublelayered $\mathrm{Pn} / \mathrm{PbPc}$ structure (just as silicon) absorbs solar light (Fig. 2, curves 1 and 2) in visible and near infrared (IR) regions, which is required for the production of SC.

Thin-film iso-type HS were prepared by sequential thermal deposition of $\mathrm{Pn}$ and $\mathrm{PbPc}$ layers on glass substrates with a conductive and semitransparent indium tin oxide (ITO) layer at different substrate temperatures $\left(T_{s}\right)$. At first, the Pn layer was deposited, and then the 


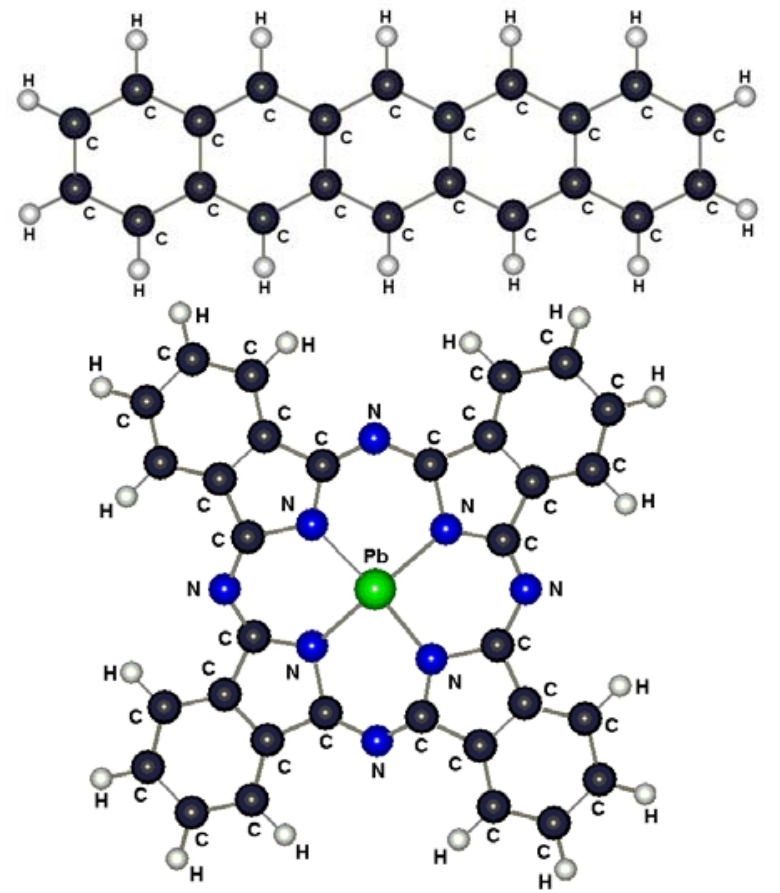

Fig. 1. Molecular structure of $\mathrm{Pn}$ (top) and $\mathrm{PbPc}$ (bottom).

$\mathrm{PbPc}$ layer was deposited on it. The thicknesses of layers were checked during the deposition by a change of the frequency of a quartz resonator and, after the deposition, by optical density spectra and atomic force microscopy and amount to about $(100 \pm 10) \mathrm{nm}$.

Absorption spectra (AS) were measured with a double-beam "Hitachi-356" spectrophotometer. The photovoltage measurements at modulated illumination were carried out by the contactless method $[8,9]$. Unmodulated monochromatic back additional illumination was created by the illumination from selected light-emitting diodes (LEDs) in the required spectral range (IR LED with the quantum energy $h v=$ $1.3 \mathrm{eV}$, whose illumination is absorbed by the $\mathrm{PbPc}$ layer, and, respectively, green LED with $h v=2.18 \mathrm{eV}$ that is absorbed by the Pn layer). The additional illumination power was measured by a calibrated radiometer PPTN-02 based on a silicon photodiode.

\section{Results and discussion}

The photovoltage in the visible and near IR ranges of iso-type HS under consideration was lower than the photovoltage of aniso-type $\mathrm{HS}$ based on $\mathrm{Pn}$ and $\mathrm{PbPc}$ layers (by 25 times for MPP/Pn HS and by 5 times for $\mathrm{MPP} / \mathrm{PbPc}$ HS [7], where MPP is methyl-substituted perylene pigment).

The feature of Pn/PbPc HS obtained at $T_{s}=300 \mathrm{~K}$ is the double reversal of a sign in the photovoltage spectra at 2.1 and $2.6 \mathrm{eV}$ (Fig. 2, curve 3). In this case, the photovoltage spectra of these HS correlate with AS of $\mathrm{PbPc}$ films in the range $1.2-1.8 \mathrm{eV}$ (actually in the

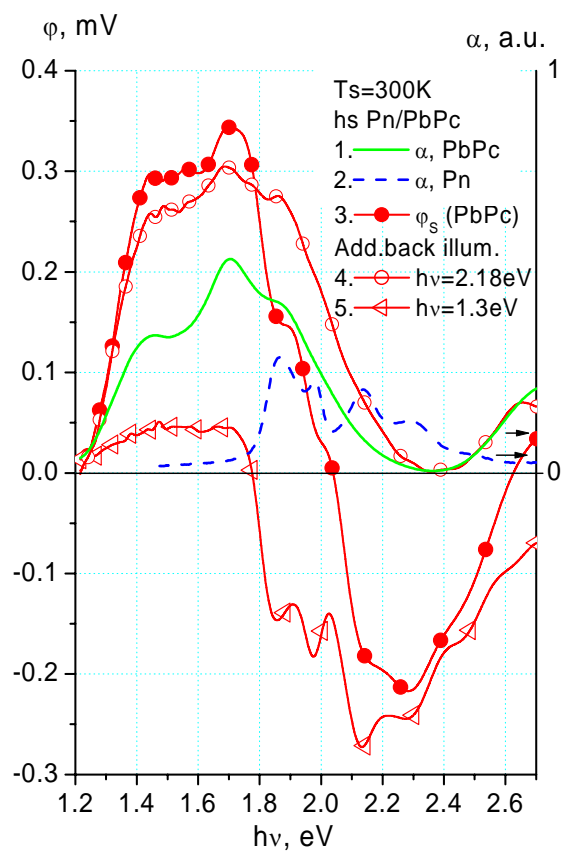

Fig. 2. Absorption spectra of $\mathrm{PbPc}(1)$ and $\mathrm{Pn}$ (2) layers at $T_{s}=300 \mathrm{~K}$ and photovoltage spectra at illumination of $\mathrm{PbPc}$ layer side for $\mathrm{Pn} / \mathrm{PbPc} \mathrm{HS}$ without (3) and with back additional illumination from LED with $h v=2.18$ (4) and $2.2 \mathrm{eV}(5)$.

transparency range of the Pn layer). In the range of significant $\mathrm{Pn}$ layer absorption (1.8-2.5 eV), the photovoltage reverse sign and the photovoltage spectra are similar to the difference of photovoltage or the absorbance of the components. According to the Van Opdorp model for inorganic iso-type HS [1], such a reversal of the sign in photovoltage spectra testifies to the formation of high-concentration surface states (centers of recombination and trapping of charge carriers) near the interface of iso-type $\mathrm{Pn} / \mathrm{PbPc} \mathrm{HS}$. These states strongly change the equilibrium profile of energy bands of the heterojunction. Charged states at the interface of two layers result in the formation of two depleted regions, and so the heterojunction can be presented in the form of two Schottky photodiodes engaged towards each other.

The model of two such Schottky photodiodes can be used not only for the description of photoelectric properties of iso-type heterostructures, but also for the explanation of the influence of the back unmodulated monochromatic additional illumination from appropriate LEDs on parameters of the diode (the component of isotype HS). Thus, the photovoltage magnitude of the negative component decreases with increase in the back additional illumination intensity with the quantum energy $h v=2.18 \mathrm{eV}$ which predominantly excites the Pn layer. The negative component disappears at the intensity of back additional illumination by 20 times higher than the basic modulated illumination (Fig. 2, curve 4), and the photovoltage spectra of HS become similar to AS of $\mathrm{PbPc}$ spectra. Vice versa, the HS photovoltage magnitude of the negative component 
increases by $20 \%$ with rise in the intensity of the back additional illumination of IR LEDs with $h v=1.3 \mathrm{eV}$ (Fig. 2, curve 5) that is absorbed by the PbPc layer. In the case of IR additional illumination, the photovoltage spectra of HS correlate with AS of Pn films, and the magnitude of the positive component decreases by more than 6 times.

In $\mathrm{Pn} / \mathrm{PbPc} \mathrm{HS}$ prepared at $T_{s}=370 \mathrm{~K}$, there is no sign reversal, and the maximal photovoltage is practically two times higher than that for $\mathrm{Pn} / \mathrm{PbPc} \mathrm{HS}$ prepared at $T_{s}=300 \mathrm{~K}$ and 10 times higher than the surface photovoltage for ITO/PbPc structures (Fig. 3). This shows that the concentration of recombination centers of charge carriers is low at the interface of isotype $\mathrm{Pn} / \mathrm{PbPc} \mathrm{HS}$ prepared at $T_{s}=370 \mathrm{~K}$. The photovoltage spectra of these HS are similar to the spectra of the absorption coefficient and surface photovoltage of $\mathrm{PbPc}$ films (Fig. 3, curves 1 and 5). But, in the region of a band with maximum at $1.85 \mathrm{eV}$, the relative photovoltage of $\mathrm{Pn} / \mathrm{PbPc} \mathrm{HS}$ is by $30 \%$ higher than the photovoltage of $\mathrm{PbPc}$ films. It is caused by the contribution of non-equilibrium charge carriers photogenerated in the Pn layer.

At the back additional illumination with $h v=$ $1.3 \mathrm{eV}$ that is absorbed by the $\mathrm{PbPc}$ layer, the photovoltage in the region of a band with maximum at $1.85 \mathrm{eV}$ becomes higher than the photovoltage of a band with maximum at $1.4 \mathrm{eV}$. This is due to a decrease of the $\mathrm{PbPc}$ contribution and illustrates the Pn contribution to the formation of the photovoltage of Pn/PbPc HS (Fig. 3, curve 2).

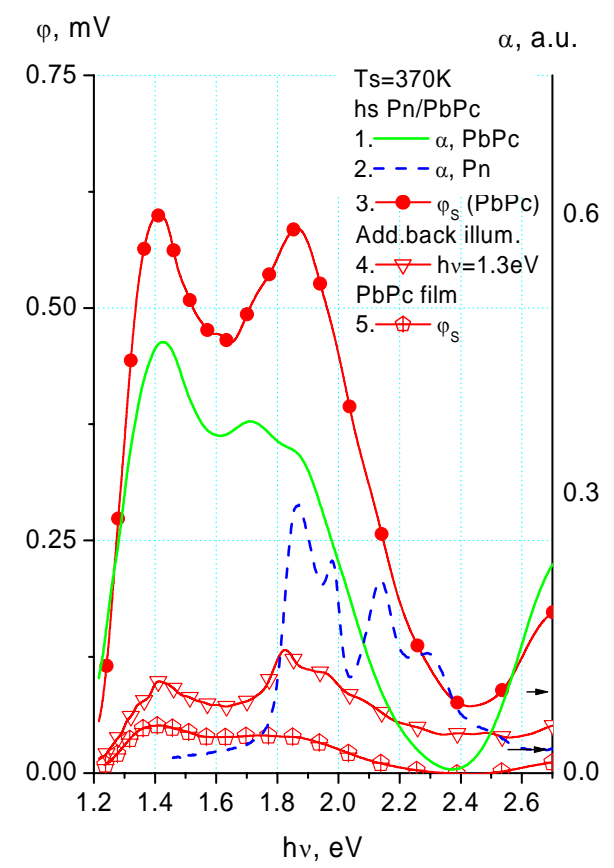

Fig. 3. Absorption spectra of PbPc (1) and Pn (2) layers at $T_{s}=$ $370 \mathrm{~K}$ and photovoltage spectra at illumination of $\mathrm{PbPc}$ layer side for $\mathrm{Pn} / \mathrm{PbPc} \mathrm{HS}$ without (3) and with back additional illumination from LED with $h v=1.3 \mathrm{eV}$ (4), and at illumination of free surface side for PbPc layers (5).
According to the Van Opdorp model for iso-type HS [1] at a high concentration of charge carriers recombination centers, the open circuit voltage (or photovoltage) can be expressed as

$$
\begin{aligned}
& \varphi=\varphi_{1} \cdot \ln \left(1+\frac{I_{R 1}}{I_{S 1}}\right)-\varphi_{2} \cdot \ln \left(1+\frac{I_{R 2}}{I_{S 2}}\right)= \\
& =\varphi_{1} \cdot \ln \left(1+\gamma_{1} \cdot P\right)-\varphi_{2} \cdot \ln \left(1+\gamma_{2} \cdot P\right),
\end{aligned}
$$

where $I_{R 1}$ and $I_{R 2}$ are the photocurrent densities through the corresponding Schottky photodiodes (i.e., diodes in corresponding HS components), $I_{S 1}$ and $I_{S 2}$ are the densities of the corresponding dark saturation currents. In this case, the corresponding $I_{R}$ is proportional to the intensity (power) of illumination $(P)$ of the corresponding $\mathrm{HS}$ components. In our case, the $\mathrm{PbPc}$ component has a positive photovoltage that corresponds to the term with index 1, and the Pn component has negative photovoltage and corresponds to the term with index 2. If the illumination is absorbed in the PbPc layer only (for example, IR light), then the dependence $\varphi(P)$ will be fitted by one term of the equation only, while if illumination is absorbed in both layers (green light), then both terms of the equation will contribute, and $\varphi(P)$ can reverse the sign. If the contribution of the second (negative) term is neutralized by an additional illumination that decreases the contribution of the corresponding component (in our case, the Pn layer contribution is decreased by the additional illumination with $h v=2.18 \mathrm{eV}$, Fig. 2, curve 4), then the basic contribution will be given by the first (positive) term.

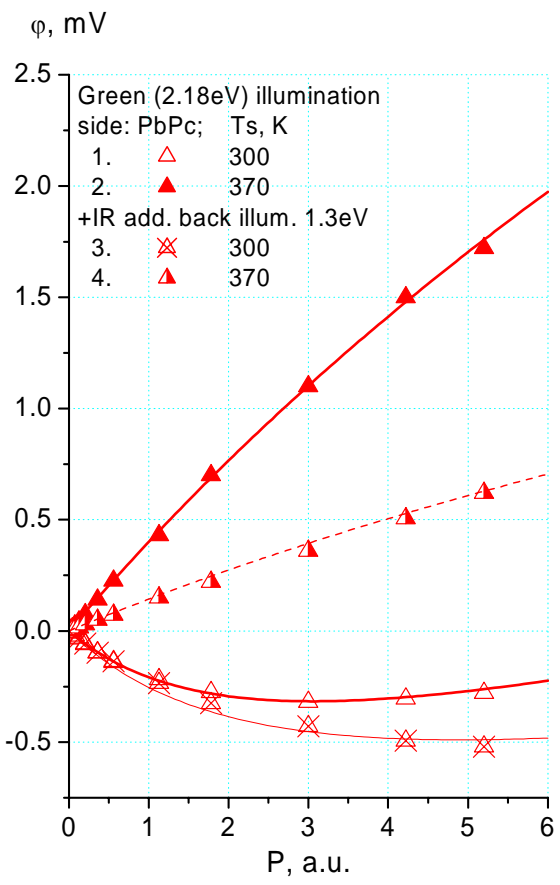

Fig. 4. Dependence of photovoltage for $\mathrm{Pn} / \mathrm{PbPc}$ heterostructure on intensity of illumination with green $(h v=2.18 \mathrm{eV})$ LED from PbPc layer side without $(1,2)$ and with $(3,4)$ back additional illumination with $h v=1.3 \mathrm{eV}$; at $T_{s}=300 \mathrm{~K}(1,3)$ and $370 \mathrm{~K}(2,4)$. 


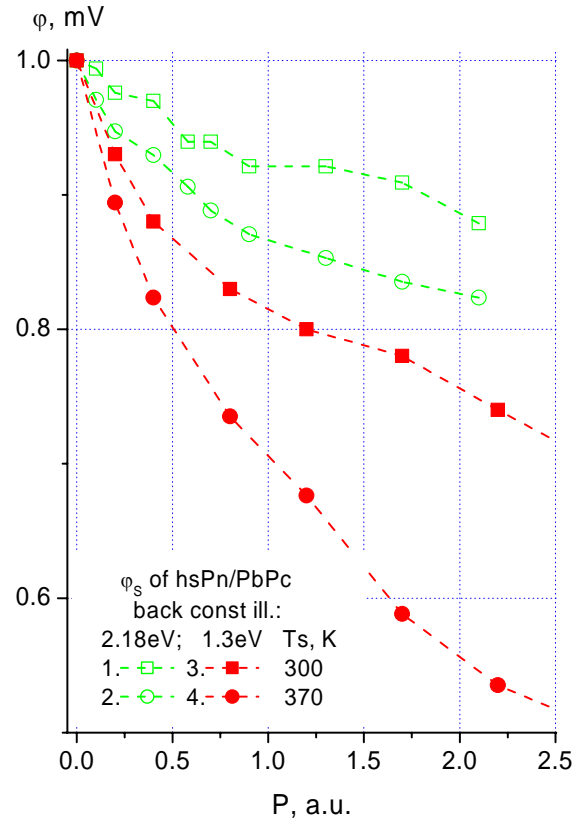

Fig. 5. Dependence of photovoltage for $\mathrm{Pn} / \mathrm{PbPc}$ heterostructure at illumination with $h v=1.45 \mathrm{eV}$ from $\mathrm{PbPc}$ layer side on intensity of unmodulated back additional illumination with green $(h v=2.18 \mathrm{eV})(1,2)$ and IR $(h v=1.3 \mathrm{eV})(3,4)$ LED; at $T_{s}=300 \mathrm{~K}(1,3)$ and $370 \mathrm{~K}(2,4)$.

To check the validity of this model in HS under investigation, we measured the $\varphi(P)$ dependences in the spectral range, where both components of HS without and with the back unmodulated additional illumination contribute to the photovoltage (Fig. 4). It is seen that the IR additional illumination, which decreases a bend of bands in the PbPc layer only, results in increasing the photovoltage in HS prepared at $T_{s}=300 \mathrm{~K}$ (Fig. 4, curves 1 and 3). In other words, the HS photovoltage components compensate each other without additional illumination, which is equivalent to the scheme of two photodiodes engaged towards. In HS prepared at $T_{s}=$ $370 \mathrm{~K}$ and with the same additional illumination, the photovoltage decreases (Fig. 4, curves 2 and 4), i.e. the photovoltage components unite without additional illumination, which is equivalent to the scheme of two photodiodes engaged in one direction [1].

The experimental dependences of $\varphi(P)$ are fitted by the proposed equation and, in such a way, have testified to the possibility of using the Van Opdorp model for organic iso-type HS (Fig. 4). In this case, the photovoltage component of the high-energy-gap layer can reverse the sign according to the expression for the short circuit photocurrent in double-layer HS with high recombination rate at the interface [1].

As is well known, the additional illumination decreases the potential barrier (a bend of bands) predominantly in the component that absorbs this additional illumination [8]. The efficiency of a decrease in the photovoltage will be higher in that component of
HS, where a change of the bend of a band is greater, i.e. the potential barrier is higher. Therefore, the distribution of space charge can be estimated at the interface of HS components with the help of the influence of an unmodulated monochromatic additional illumination on the photovoltage. The additional illumination with $h v=$ $1.3 \mathrm{eV}$ is absorbed in the PbPc layer only, and one with $h v=2.18 \mathrm{eV}$ is absorbed predominantly in the Pn layer. It is shown in Fig. 5 that the additional illumination with $h v=1.3 \mathrm{eV}$ more strongly affects the photovoltage of $\mathrm{Pn} / \mathrm{PbPc} \mathrm{HS}$ than that with $h v=2.18 \mathrm{eV}$. However, it should be noted that both additional illuminations affect the photovoltage more strongly in HS prepared at $370 \mathrm{~K}$. According to the above results, the space charge is basically localized in the PbPc layer for both types of structures, and the bend of bands at the interface of HS is greater for structures prepared at $370 \mathrm{~K}$.

\section{Conclusions}

The obtained data allow us to consider that, at the interface of organic iso-type $\mathrm{Pn} / \mathrm{PbPc}$ heterostructures prepared by thermal deposition on substrates at a temperature of $300 \mathrm{~K}$, the high concentration of the recombination centers of charge carriers is formed. As a consequence, the sign reversal is observed in photovoltage spectra of these heterostructures. These structures are described within a model of two photodiodes engaged towards which proposed by Van Opdorp for inorganic heterostructures. In this case, the negative photovoltage component decreases at the excitation of the Pn layer by the unmodulated additional illumination with $h v=2.18 \mathrm{eV}$ and, on the contrary, increases at the excitation of the $\mathrm{PbPc}$ layer by the unmodulated additional illumination with $h v=1.3 \mathrm{eV}$.

There is no sign reversal of photovoltage spectra for $\mathrm{Pn} / \mathrm{PbPc} \mathrm{HS}$ prepared at $370 \mathrm{~K}$, and the photovoltage in the range of strong absorption is significantly higher than the photovoltage of HS prepared at a substrate temperature of $300 \mathrm{~K}$. This indicates that the recombination rate of charge carriers at the interface of $\mathrm{Pn} / \mathrm{PbPc}$ heterostructures decreases with increase in the substrate temperature from 300 to $370 \mathrm{~K}$. Therefore, the iso-type $\mathrm{Pn} / \mathrm{PbPc}$ heterostructures prepared at $370 \mathrm{~K}$ allow one to widen the spectral range of the sunlight absorption, to improve the coefficient of charge carrier collection, and, as a result, can be used for increasing the efficiency of organic solar cells.

\section{References}

1. B.L. Sharma, R.T. Purohit, Semiconductor Heterojunction. Pergamon Press, Oxford, UK, 1974.

2. Ya.I. Vertsimakha, Organic iso-type heterostructures and prospects of their practical application for organic solar cells // Abstracts $4^{\text {th }}$ Intern. Confer. on "Electronic Processes in Organic Materials" (ICEPOM-4) June 3-8, 2002, Lviv, Ukraine, p. 45-46 (2002). 
3. A.K. Pandey, J.-M. Nunzi, Efficient flexible and thermally stable pentacene $/ \mathrm{C}_{60}$ small molecule based organic solar cells // Appl. Phys. Lett. 89, p. 213506 (2006).

4. C. Hamann, H.-J. Höhne, F. Kersten, M. Müller, F. Przyborowski, M. Starke, Switching effects on polycrystalline films of lead phthalocyanine $(\mathrm{PbPc})$ // Phys. status solidi (a) 50, p. K189-K192 (1978); K. Ukei, K. Takamoto, E. Kanda // Phys. Lett. A 45, p. 345 (1973).

5. Ch.-K. Song, M.-K. Jung, B.-W. Koo, Pentacene thin film transistor improved by thermal annealing // J. Korean Phys. Soc. 39, p. S271-S274 (2001).

6. J. Puigdollers, C. Voz, A. Orpella, I. Martin, M. Vetter, R. Alcubilla, Pentacene thin-films obtained by thermal evaporation in high vacuum // Thin Solid Films 427, p. 367-370 (2003).

7. Ya. Vertsimakha, P. Lutsyk, $p$ - $n$ type heterostructures based on N, N`-dimethyl perylenetetracarboxylic acid diimide // Molec. Cryst. Liquid Cryst. 467, p. 107-122 (2007).

8. I.A. Akimov, The investigation of internal photoelectric effect in semiconductors by condenser method // Optiko-Mekhanicheskaya Promyshlennost' 5, p. 4-13 (1966) (in Russian).

9. P. Lutsyk, J. Misiewic, A. Podhorodecki, Ya. Vertsimakha, Photovoltaic properties of $\mathrm{SnCl}_{2} \mathrm{Pc}$ films and $\mathrm{SnCl}_{2} \mathrm{Pc}$ /pentacene heterostructures // Solar Energy Mater. Solar Cells 91, p. 47-53 (2007). 